\title{
Design and Implementation of A Patient-Doctor Monitoring System: A Review and A Telemedical Algorithmic Approach for Non-Invasive Post-Malaria-Diagnosis Monitoring
}

\author{
Benjamin Kommey, Akwasi Darkwah Akwaboah, Josephine Owusu-Akyaw
}

\begin{abstract}
In an era where there is a globally concerted effort to improve healthcare especially in regions with endemic diseases like Sub-Saharan Africa, there have been major calls for real-time health data acquisition and reporting[1] to prompt health institutions and government to adequately plan and tackle such challenges. Data Acquistion is also very important as it will in the long run drive other future research work. Due to these eminent reasons, this paper seeks to review a number of situational reports, relevant projects and papers with proposed solutions or implemented prototypes that can be employed in monitoring the recovery and health status of patients that are diagnosed of Malaria. This paper also proposes an operational algorithm (based on the existing projects' review) of a wearable embedded device in the form a flowchart to inspires device makers and innovators to design systems and devices to help in the fight against Malaria, which currently claims the lives of thousands annually. [2] The focus, though on Malaria because of its prevalence in the SubSaharan Africa, can be employed on other similar endemic diseases
\end{abstract}

Keywords—-monitoring, HealthCare, malaria, telemedicine, algorithm, device.

\section{INTRODUCTION ${ }^{1}$}

$\mathrm{T}$ The use of telemedicine in health monitoring and realtime data collection of the health status of individuals, particularly, in disease endemic regions like sub-Saharan Africa and other developing nations is very vital in winning the fight against endemic diseases like malaria, measles, rotavirus and child bacterial meningitis. Even though, significant strides have been attained over the past decade in arriving at quality data collection, there is still more room for improvement to enable accurate and timely data reporting [1]. With such data gathered by a telemedicine-based technology, good data driven decisions can be made which will eventually inform attending doctors and even leadership of health institution and nations how to strategize against such diseases.

It is in this light, that this paper seeks to review existing technology that address this challenge and subsequently propose an alternative solution in the form of an algorithm. This alternative named PatDocSpy involves employing a wearable embedded device to measure vitals of post-malaria diagnosed patients with the aim of monitoring recovery. This is integrated with a mobile app platform that receives data from the data acquisition device (DAQ) and transmits to the attending medical doctor. A chat system could also be incorporated to allow real time communication between the patient and the Doctor.

\footnotetext{
Benjamin Kommey, Akwasi Darkwah Akwaboah, Josephine OwusuAkyaw are with Departement of Computer Engineering, Kwame Nkrumah University of Science and Technology, PMB KNUST, Kumasi, Ghana. Email: bkommey.coe@knust.edu.gh; adakwaboah@gmail.com; phinna9@yahoo.com.
}

\section{LITERATURE REVIEW}

\section{A. Malaria}

Malaria is an infectious disease caused by the presence of the plasmodium parasite in its (human) host. This disease is endemic in Central America, northern regions of South America, South and Southeast Asia and greatly in Africa (specifically Sub-Sahara), Symptoms include include fever, anaemia, malarial paroxysm (rigor, ie. Sudden feeling of coldness accompanied by shivering and increased body temperature, as high as $39^{\circ} \mathrm{C}$ to $41^{\circ} \mathrm{C}$ and profuse sweating), polyuria, ie. Increased urine production/ urination, severe headaches, nausea, rapid and thready pulse and inactivity due to extreme fatigue [2].

\section{B. Reviews}

This section contains definition of certain key terms and a review of situational reports, several related projects and inventions existing which the aim of identifying room for possible modifications and improvements to these existing technologies.

In medical terms, Monitoring deals with the observation of a disease, condition or one or more medical parameters over time. Monitoring generally seeks to provide information on a trend in patients' performance or response to treatment.

A review of situation reports driving the need for such a technology has been done. Also, a number of related projects, inventions have been done in trying to arrive at a reliable telemedical health monitoring system, some of which are reviewed here.

1. The African Regional Health Report presents a situational report on the state on health in Africa and feasible recommendation to augment already existing attempts to address challenges in the African health sector [1]. It places emphasis on proposed indices for 


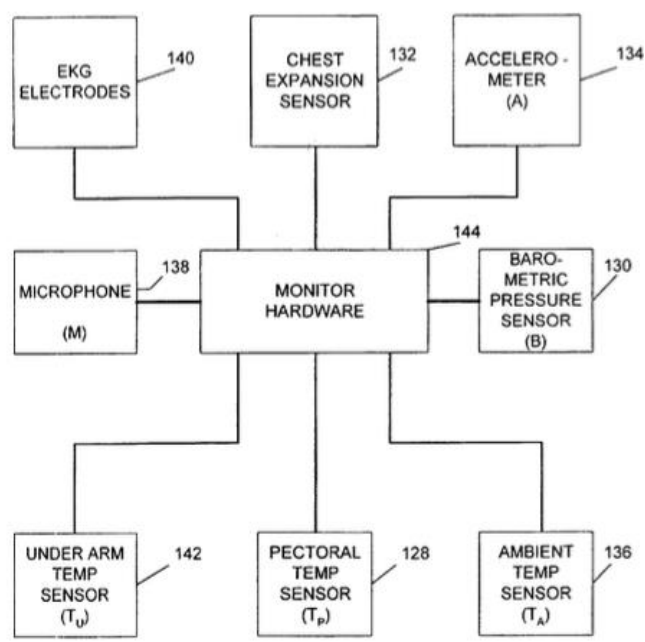

Figure 2. Organization of data collection component.

ensuring a thriving health sector, ie. Good governance for health, policy formulation on health, quality assurance and most importantly data-driven decision making, which is the motivation behind this project. It outlines the relevance of timely and accurate data collection and reporting. One notable instance when early detection of disease symptoms in cases such as Buruli Ulcer, dracunculiasis, human African trypanosomiasis, leprosy, leishmaniosis, yaws among others, can make a life-saving difference. More so, the report recommends the adoption of "low-cost health solutions, particularly prevention and health promotion, for the entire population" as the way forward for addressing the technological divide between Africa and the west [1]. It also emphasizes on the need to expand real-time data collection and reporting "to provide accurate data informing action on all the significant threats to human health in the Region" [1]. This points to the important of innovating in health monitoring as there is a need for it in the Region.

2. The World Malaria Report 2017 elucidates the global state of malaria prevalence with particular emphasis on preventive and diagnostic methods and corresponding statistics. It also presents information on the regional and global trends in Malaria cases with a staggering 445,000 reported Malaria-related deaths in 2016, out of which Africa contributes $90 \%$ of total reported cases and death with $80 \%$ of this percentage being as a result of cases from Sub-Sahara Africa [3].

3. In the health monitoring invention of Raymond et al, a system comprising of three basic components: (a) A data management system (b) a plurality of physiological and subjective data collection devices that collects a set of timestamped serial streams from a subject (c) A communication system for data transferred between a Real Time(RT) microcontroller and a Memory Server (MS) microcontroller (Figure 1) [4]. One key feature of this invention is the implementation of a multipara metric monitor as an incorporation of the data collection component that comprises of a chest strap with distribution of various sensors and supporting electronics with flexible conductor connections. The measured parameters

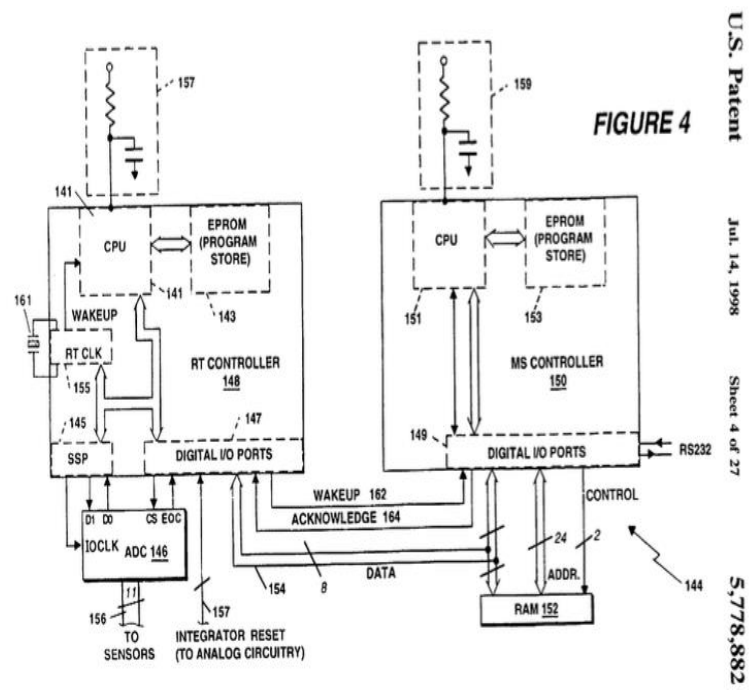

Figure 1. Real Time (RT) \& Memory Server (MS) Microcontrollers.

include body temperature, breathing rate (ventilation), ambient temperature measurement, atmospheric pressure and heart activity. In addition to this, a data logger to receive subjective data is added (Figure 2). The data management system, or to a large extent the MS microcontroller, primarily sorts the collected data and saves into appropriate memory location. The memory is divided into three blocks; (a) the scratchpad for storing data temporarily (b) the warehouse for all but 8-bit EKG data storage. (c) the 8-bit EKG area. When necessary the data is compressed as the system awaits retrieval by the health professional or the user. The data results from this invention is not tied to any particular disease or disorder but rather to the general wellbeing of the subject of interest. In addition to the abovementioned feature, a detailed flowchart of parametric data processing is included in the paper. Possible improvements for this invention is a fusion of the two microcontrollers into one to provide economic benefits and simplicity of design. Also additional parameters can be measured.

4. In the work of Ch. Sara, a real-time health monitoring system [5] has been designed which reminds the patient to take medication and reports vital signs and frequency of medication intake. It also tracks and report the position of the patient using GPS to inform the doctor of the patient's activity. This system was built using ARM7 microcontroller and consists of three components - a central health-IOT system called iMed box, iMed pack and Bio_patch. The iMed box sends information regarding patient to the doctor through the internet and also reminds the patient to take medication at prescribed timings. The iMed box coordinates the Bio patch and iMedpack which are connected to it. The Bio patch on the other hand is employed in continuous monitoring of the patient and then forwards acquired information to the iMed box for transmitted to the Doctor.

5. Sagahyroon worked on a wearable healthcare monitoring system [6] that essentially measure vital signs via sensor-microcontroller integration and transmits these readings via Bluetooth to the patient's mobile phone and sends an SMS alert to the Doctor in emergency cases. The mobile app upon receiving 
patient's vital signs, also updates the patient records in a hospital database.

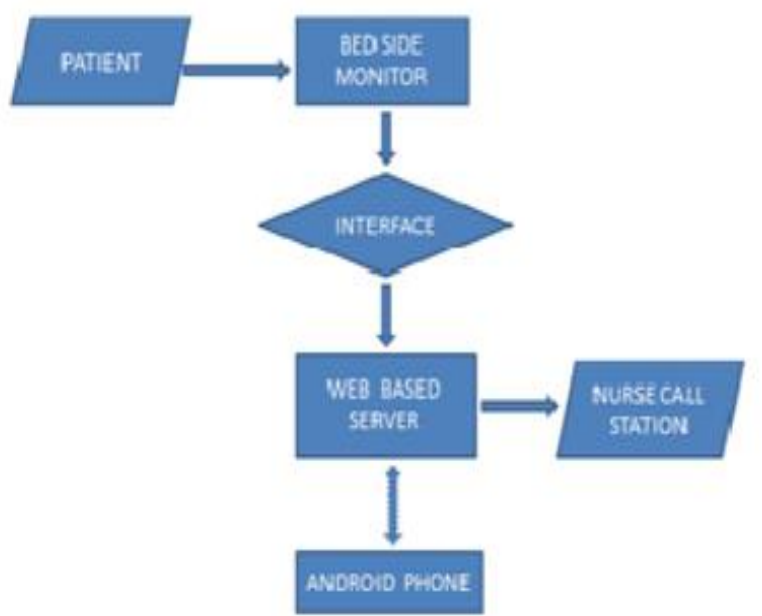

Figure 3. Block diagram of android based telemedine project.

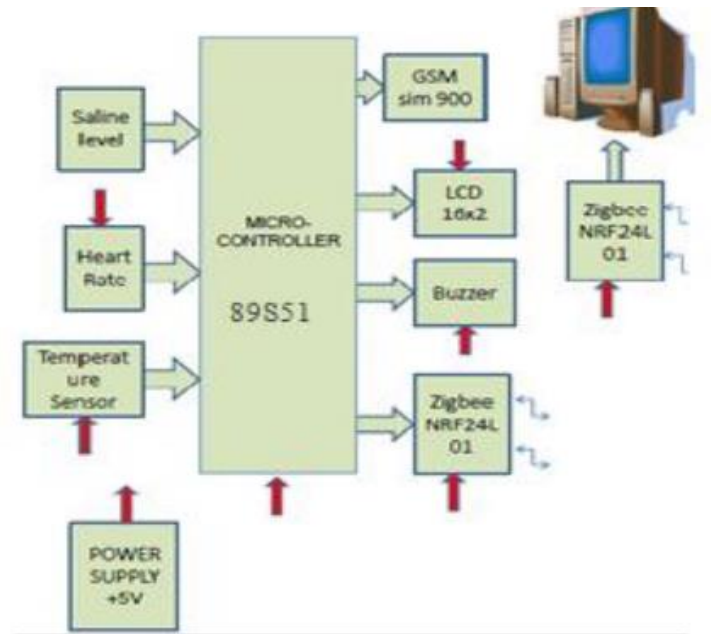

Figure 4. System block diagram of the wireless family health monitoring system.

6. According to Preman Sundaram, Telemedicine is an aspect of medicine where medical information is transferred through a wireless network from one point to the other (usually from a patient to a doctor and vice versa) for the purpose of consulting, monitoring and delivering of many diverse medical services [7]. In telemedicine, the doctor or medical practitioner need not be at the bedside of the patient to monitor the patient's progress and vital signs and in some cases offer timely interventions. Monitoring devices used in telemedicine can be used to monitor information like blood pressure, heart rate, blood glucose, hemoglobin, oxygen saturation among many others. Remote patient tele monitoring aids in keeping track of patients outside the hospital setting which makes this system less costly (Figure 3). The control panel is used to create the database. The main software used is XAMPP. The aspect of XAMPP that was used to create the database in this project was Apache and MySQL. There is a patient details window which is used to create the database containing the details of the patient (patient id, name, mobile number, address, age, gender and feedback). Provision is made available to update the database.

7. Also, in the monitoring system produced by Sunil Patil, medical practitioners can monitor and examine

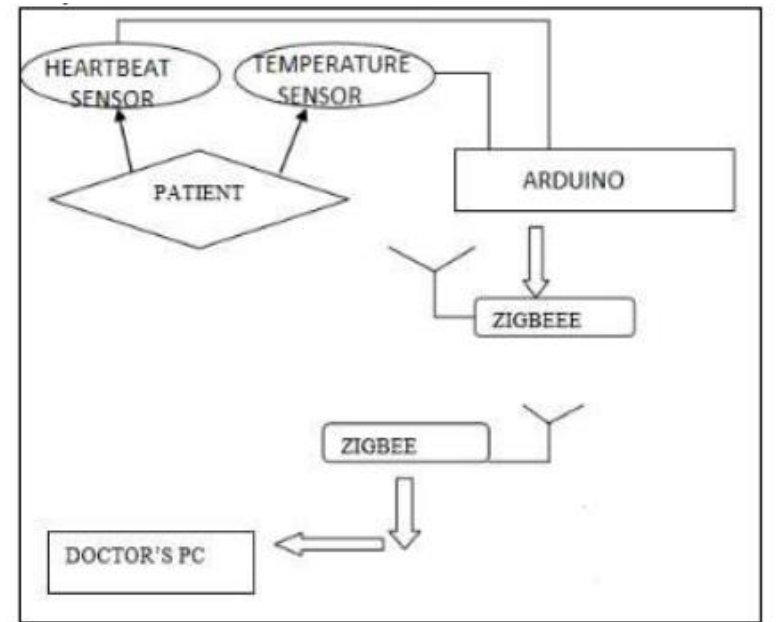

Figure 5. Block diagram of operation of patient monitoring to remote doctor project.

TABLE 1.

MALARIA SyMPTOMS WITH THEIR MEASURABLE PARAMETERS \& DEGREE OF INVASIVENESS

\begin{tabular}{ccc}
\hline \hline Symptom & $\begin{array}{c}\text { Measurable } \\
\text { Parameter(s) }\end{array}$ & $\begin{array}{c}\text { Categorization by } \\
\text { Invasiveness of measurement }\end{array}$ \\
\hline Fever & Body Temperature & Non-invasive \\
Anaemia & Comoglobin $(\mathrm{Hb})$ & Invasive \\
& Body Temperature & Non-invasive \\
& Pulse Rate & Non-invasive \\
Malaria & Sweat/ Skin & Non-invasive \\
Paroxysm & Moisture level & - \\
& Headache & - \\
Polyuria & Nausea & Non-invasive \\
Extreme & Activity Level Collection & Non-invasive \\
Fatigue & &
\end{tabular}

patient critical health parameters using Zigbee technology. Here, the doctor or healthcare provider can be contacted or alerted in case of emergency through Short Message Service (SMS) [8]. This offers the patient full mobility at home while the health information being remotely assessed by the healthcare provider. This system can only be used for immobile (admitted) patients [8]. The system uses three sensors, that is temperature, heartbeat and saline level sensors. The system can help a doctor to diagnose or monitor remotely through the internet (Figure 4). System block diagram where patient's saline level, heart rate and temperature are taken by wireless sensothe patients heartbeat is wirelessly transmitted to the microcontroller through Zigbee. It uses LM358 which is an OPAMP being used as a comparator. The Gsm modem accepts a sim card and operates just like a mobile phone with its own mobile number. How it works: whenever an abnormality is detected by the sensors, a message is sent to the the designated mobile phone through the GSM modem which is then given to the Zigbee module and the appropriate message will be displayed on the LCD and also on the computer.

8. Shivam Gupta et al present a development of a microcontroller based system for wireless heartbeat and temperature monitoring using wifi module [9]. 
TABLE 2 .

INVASIVE PARAMETERS \& THEIR SENSING MECHANISM

\begin{tabular}{ccc}
\hline \hline Parameter & Proposed Sensor/Sensing Mechanism & Sensor Description \\
\hline Body Temperature & LMT70[10] & $\begin{array}{c}\text { This is a Texas Instruments ultra-small } \\
\text { precision, low-power cmos analog temperature } \\
\text { sensor with +/- 0.1C precision. It is best suited } \\
\text { for medical applications like Human skin } \\
\text { temperature sensing. Eg. TIDA-00824 [11] }\end{array}$ \\
Skin Moisture (Sweat) & Galvanic Skin Response (GRS) Sensor & - \\
Pulse Rate & Pulse/ Heart rate sensor & The degree of randomness in accelerometer \\
Activity Level & Accelerometer & measurements will indicate activity level \\
\hline \hline
\end{tabular}

9. In Dr. B. V. Sumangala's project report on a wireless remote health monitoring system, a ZigBee module was used in wireless data transmission. Because Zigbee has low power consumption, it is usually used for 24-hour monitoring of communication transmission sytems. Compared to Bluetooth, Zigbee has large number of node which allows for future expansion of the systems, higher network flexibility and better transmission range. [12] An LM35 temperature sensor and pulse sensors have their inputs connected to the patient and their output pins connected to the ADC pins of an Arduino Mega 2560 microcontroller. The next part of the communication is the transmittion and reception parts which make use of two separate zigbees. The transmitter zigbee is connected to the microcontroller/Arduino and the receiver zigbee is connected to the doctor's pc. At the transmitting end, the sensors output is received by the Rx pin of the Transmitting zigbee and is then sent through the Tx pin of the Arduino. The data is then transmitted to the reception end wirelessly via the end zigbee connected to the doctor's pc. The two zigbees identify each other by having the same PAN ID. The Arduino is programmed to aid in the systems monitoring (Figure 5).

\section{METHODOLOGY}

The proposed solution is an idea that entails a wearable embedded device with the aesthetics of a wrist watch that could be given to users post malaria diagnoses (during recovery), and with their consent to be worn as often as possible with the aim of obtaining monitoring information. This information will then be sent to the mobile phone of the patient which in turn transmits the information to the attending medical doctor. The wearable device will

1. Measure subject's body parameters which in this case are body surface temperature and moisture levels, pulse rate and activity level. Other parameters could also be added

2. Measure ambient parameters; temperature and humidity

3. Compare body parameters and ambient parameters and make inferences by either relating measured body parameters to measured ambient parameters or to disease

4. Find relation between the activity level and the body parameters.
5. Check for and establish wireless (Bluetooth) connectivity, and Upload data to the mobile phone.

\section{A. Measurands}

Body surface temperature and moisture levels were chosen, because their abnormal levels are indicative of symptoms of fever which are generally linked to Feverbased diseases like malaria (reference). Also, the pulse rate and activity levels are used to determine whether the subject's changes in heart rate is as a result of physical activity. These four parameters ie., body surface temperature, moisture (sweat) levels, activity level and pulse rate were chosen because they provide a noninvasive way of monitoring state of malaria-diagnosed patient per the corresponding symptoms in section II.A.

Table 1 has information on the various symptoms of malaria with corresponding measurable body parameters with categorization on whether measurement of each parameter is by either invasive or non-invasive means. Table 2 contains descriptions of the four non-invasive parameters under study and a table of the various parameters and their corresponding sensors/ sensing mechanism.

In trying to relate low activity level to disease, conclusion should reached in the context of the ambient conditions and subject's body temperature. As a person engages in rigorous exercise, the body temperature rises due to heat produced as a by-product of oxygen combining with ATP to create energy. The body however needs to regulate the body temperature as soon as possible. Internal temperature sensors therefore signal the hypothalamus to reduce the body temperature.

Hence the body temperature increases during exercise for some time until the hypothalamus can efficiently cause it to reduce to the normal body temperature.

The heart rate and the pulse rate are the same.

having a heart rate that exceeds the normal range (60$100 \mathrm{bpm})$ is known as tachycardia - a heart rate that exceeds the normal resting rate. Tachycardia may be caused by rises in body temperature, exercise, stress and heart disease [11].

From the above it can be inferred that, exercising in a way increases body temperature both of which can lead to tachycardia. (increased pulse rate). This body temperature can then be measured using a temperature sensor as shown in Figure 6.

\section{B. The Algorithm.}

The Operational Algorithm for the proposed wearable device to serve the purpose of a DAQ is shown in figure 


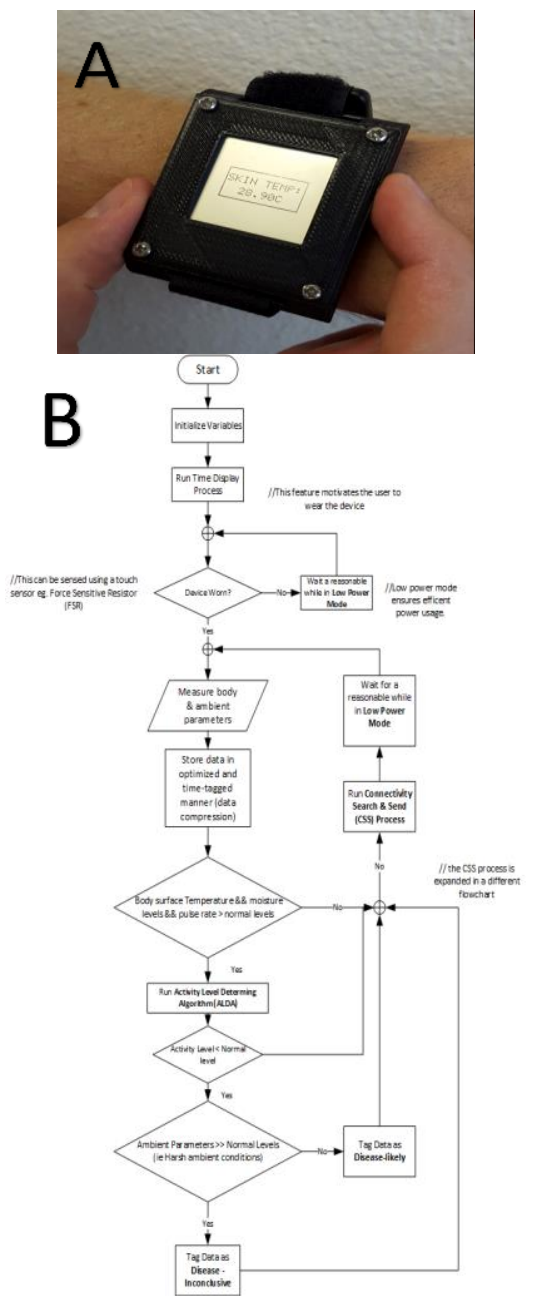

Figure 6. (a) A skin temperature sensor: Credit (http://www.ti.com/tool/TIDA-00824), (b) Flowchart/ operational algorithm of proposed wearable device.

6. On start, the device initializes all variables to set constant and on-start variables to be set to the appropriate values. Next, the time display process which displays time on the device's screen. This is to give the device an add-on time telling feature to encourage the user to wear it. After this, the device checks whether the user has it worn. This check could be done with a force sensitive resistor, mechanical sensing or other appropriate tactile sensing mechanism. The rationale behind this to ensure data acquisition is only carried out when device is worn so power and memory space are not unnecessarily wasted.

The Body parameters which include the body surface temperature and moisture (sweat) levels and pulse rate.

These parameters are then checked for abnormal levels and give some information on disease state but generally vague. Therefore, the Activity Level Determining Algorithm (ALDA) is run to gather more informative data for a relatively better conclusive analysis. The ALDA show in Figure 7 employs the use of standard deviation on the sampled accelerometer readings which gives an indication how active or inactive the subject is. A high standard deviation means high activity level and results are most likely to randomized by subject's movements. If the activity level is also found to be below normal levels and other parameters at higher than normal levels. The patient's recovery or state can be considered to be in bad or worse state depending on the degree of

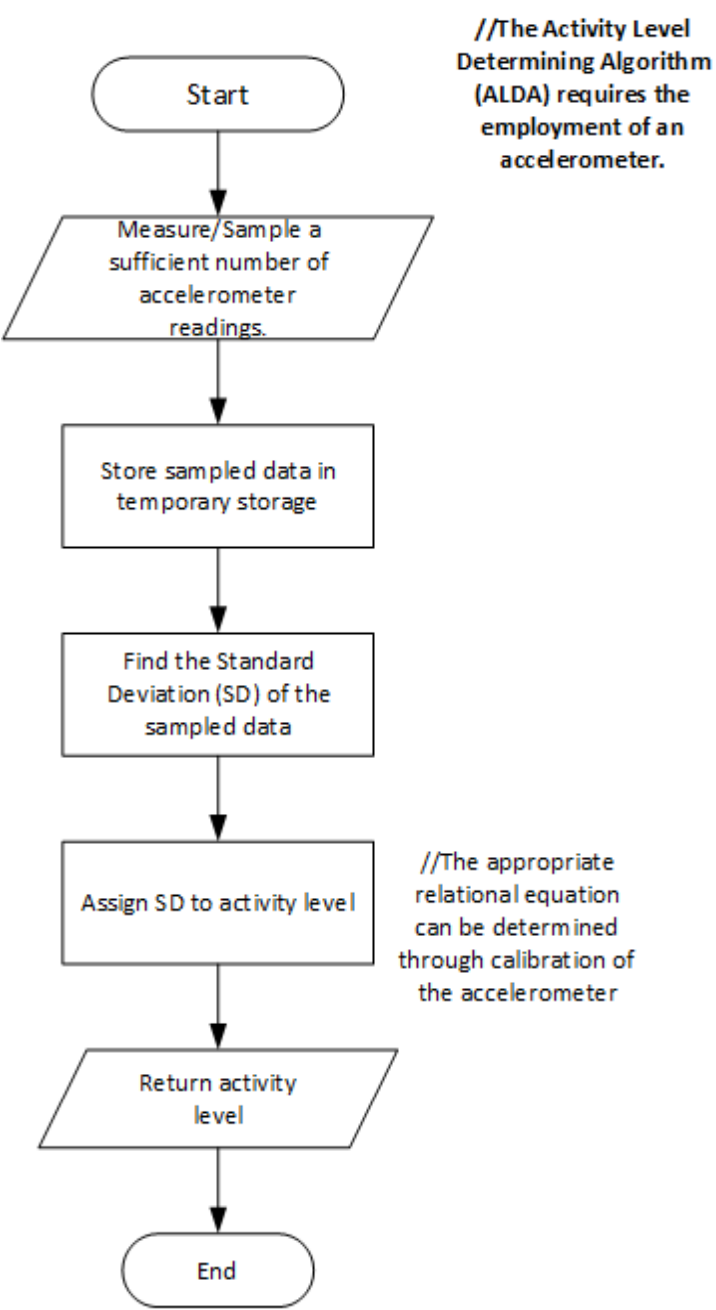

Figure 7. Activity Level Determining Algorithm (ALDA) Flowchart.

the readings and vice versa if parameter results are opposite what are mentioned above.

Nevertheless, at various stages of the device operation, measurements are transmitted to the subject's mobile device via short-range wireless communication (eg. Bluetooth) to be forwarded later to the health professional by a mobile application. The doctor may then respond to the patient through a real-time chat system on the mobile app.

\section{DISCUSSIONS}

According to the World Health Organization (WHO) Global Technical Strategy for Malaria (GTS) [12] sets four (4) ambitious but reachable targets for 2030;

1. Reducing Malaria case incidence by at least $90 \%$

2. Reducing Malaria mortality rates by at least $90 \%$

3. Eliminating in at least 35 countries.

4. Preventing a resurgence in all countries that are malaria free.

In addition to the GTS, the Sustainable Development Goals (SDGs) Goal 3 Target 3.3, the eradication of malaria, HIV/AIDS, tuberculosis and other neglected tropical diseases is captured. The Success of this SDG is strongly tied to that of the GTS.

The employment of technology in ensuring the realizing of these goals especially on the African continent is crucial. This is the motivation behind this paper, with a 
focus on Malaria monitoring and real-time data reporting in an efficient and convenient manner. Furthermore, the intention is to encourage innovation among healthcare stakeholders from health professionals, researchers, device manufacturers, etc. This paper also serves as a pointer to past projects from which makers can draw inspiration.

\section{REFERENCES}

[1] D. Kebede and et al, "The Health Of The People, The African Regional Health Report 2014," The World Health Organization Regional Office for Africa, 2014. [Online]. Available: http://www.who.int/bulletin/africanhealth/en/ .

[2] M. H. Beers and et al, "The Merck Manual of Diagnosis and Therapy," 2006

[3] World Health Organization, "World Health Malaria Report 2017." [Online]. Available: apps.who.int/iris/bitstream/10665/259492/1/9789241565523eng.pdf .

[4] Raymond and et al, "Health Monitoring System," 1998.
[5] C. Sara and et al, "Design and Implementation of Health Monitoring System using ARM7," IEEE Xplore Digit. Libr.

[6] Sagahyroon and et al, "Design and Implementation of a wearable healthcare monitoring system," National Center for Biotechnology Information, 2009. .

[7] P. Sundaram, "Patient Monitoring System Using Android Technology," Int. J. Comput. Sci. Mob. Comput., vol. 2, no. 5, pp. 191-201, 2013.

[8] S. Patil and et al, "Wireless Family Health Care Monitoring System," Int. J. Eng. Tech. Res., vol. 3, no. 2, pp. 90-92, 2015.

[9] S. Gupta and et al, "IOT Based Patient Health Monitoring System," Int. Res. J. Eng. Technol., vol. 4, no. 3, pp. 2316-2319, 2017.

[10] Anonymous, "LMT70, Precision Analog Temperature Sensor Datasheet." [Online]. Available: http://www.ti.com/product/lmt70.

[11] Texas Instruments, "Human Skin Temperature Sensing for Wearable Appliations Reference Design.” [Online]. Available: http://www.ti.com/tool/TIDA-00824.

[12] World Health Organization, "Malaria: Global Targets \& Global Technical Targets for Malaria: 2016-2030,” 2015. 